L'auteure évoque un jugement de la Cour de justice de I'Union européenne qui mérite d'être relevé. Les postulats qu'elle en dégage pour la Loi sur les produits thérapeutiques en Suisse et pour les réseaux de soins intégrés dans la LAMal sont à mes yeux justifiés: il est difficile d'inventer des incitatifs financiers intelligents, que ce soit entre médecins et industrie ou médecins et caisses-maladie ils devraient donc au moins être transparents. Une remarque supplémentaire: les banques nous ont démontré qu'également les systèmes incitatifs internes aux entreprises recèlent un immense potentiel destructeur pour les clients concernés*; la transparence est donc nécessaire aussi au sein des caisses-maladie. Les citoyens ont le droit de savoir si tout ce qu'on entend de source généralement bien informée est exact, à savoir que des assureurs-maladie verseraient des bonus aux case managers en fonction de leur résultat - c'est-à- dire que les employés d'une caisse-maladie toucheraient un meilleur salaire s'ils coupent davantage dans les prestations à rembourser aux assurés dont ils ont la charge.

Hanspeter Kuhn, avocat, secrétaire général adjoint de la FMH

* Cf. Roth M. Wenn der Berater in Tat und Wahrheit Verkäufer ist. IFZ Blog. News aus dem Institut für Finanzdienstleistungen Zug IFZ. 5. 8. 2010. Emmenegger S. Anlagekosten: Retrozessionen im Lichte der bundesgerichtlichen Rechtsprechung In: Emmenegger S (Hrsg). Anlagerecht. Basel: Helbing Lichtenhahn; 2007. Felber M. Streit über die Regulierung von Retrozessionen. NZZ 12. März 2010: Retrozessionen, Provisionen und ähnliche Vermittlungsvergütungen im Finanzsektor werden in der Schweiz und der Europäischen Union (EU) zunehmend reguliert. Dies soll Interessenkonflikte beim Vertrieb von Finanzprodukten begrenzen.

\title{
La liberté thérapeutique du médecin face aux incitatifs financiers ciblés de l'Etat
}

Valerie Junod

Professeur à la Faculté des hautes études commerciales (HEC) de l'Université de Lausanne et à la Faculté de droit de l'Université de Genève

L'auteur remercie ses trois relecteurs habituels pour leurs commentaires.

Correspondance: Prof. Dr Valerie Junod HEC

Internef 615

Université de Lausanne CH-1015 Lausanne

valerie.junod@unil.ch
The pure and simple truth is rarely pure and never simple. (The Importance of Being Earnest by Oscar Wilde)

L'Etat peut-il délibérément influencer la liberté de prescription des médecins par des incitations financières? La Cour de justice de l'Union européenne (la Cour) a récemment répondu «oui», dans une affaire anglaise qui présente également un intérêt pour la Suisse [1].

\section{Comment concilier budgets étatiques et indépendance des médecins?}

La Cour devait trancher la plainte élevée par l'association faîtière de l'industrie pharmaceutique britannique (ABPI) à l'encontre du gouvernement anglais. Ce dernier, par l'intermédiaire des caisses de soins primaires, attribue une rémunération spéciale aux médecins qui acceptent de prescrire certains médicaments bon marché plutôt que d'autres plus chers [2]. Il ne s'agissait en l'occurrence pas d'une simple substitution générique, mais d'une substitution au sein de classes thérapeutiques. L'ABPI défendait le point de vue qu'un tel versement aux médecins violait l'article 94 de la Directive 2001/83 [3], une disposition similaire à notre fameux article 33 de la Loi fédérale sur les produits thérapeutiques (LPTh) [4] qui interdit la promesse et l'acceptation d'avantages matériels.
Pour l'ABPI, l'interdiction d'octroyer des avantages matériels aux médecins sert à préserver leur indépendance et leur objectivité au moment de choisir le médicament le mieux adapté à leurs patients [5]. La mesure étatique en cause biaise directement ce choix, en incitant les médecins à opter pour le médicament le moins cher. Selon l'ABPI, l'interdiction érigée à l'article 94 devait s'appliquer aussi bien à l'industrie pharmaceutique qu'à l'Etat lui-même.

Alors même que la Commission européenne et l'Avocat général auprès de la Cour s'étaient rangés du côté de l'ABPI [6], la Cour a rejeté leurs arguments. Bien qu'un arrêt récent ait tranché que les règles de la Directive 2001/83 sur la publicité ne s'appliquent pas qu'aux sociétés pharmaceutiques, mais aussi aux tiers indépendants (par ex. un journaliste sans lien avec l'industrie, comme dans l'affaire Damgaard [7]), la Cour a refusé de franchir le pas supplémentaire en rendant ces règles applicables aux gouvernements. $\mathrm{Au}$ contraire, les autorités nationales doivent pouvoir continuer à élaborer et à appliquer leurs règlementations, y compris en définissant «les priorités d'action de la politique de santé publique, en particulier en ce qui concerne la rationalisation des dépenses publiques». Parce que définir de telles politiques de santé ne poursuit «aucun but lucratif ni commercial», il leur est permis de recourir à des incitations financières accordées aux médecins, sans qu'on se trouve 
«dans le cadre de la promotion commerciale de médicaments». Pour la Cour, l'article 94 de la Directive européenne ne vise que des actions qui se situent dans un tel cadre; hors de ce cadre, les Etats membres et leurs caisses agissant sur délégation - conservent leur marge de manœuvre.

La Cour complète ce raisonnement par trois arguments. D'abord, les mesures d'un Etat pour orienter la prescription ne sauraient, contrairement à celles d'entreprises ou de personnes privées, créer un risque pour la santé publique, car la mission même des autorités est «de veiller à cette santé publique, pour laquelle elles assument la responsabilité politique.» Ensuite, les médecins doivent être capables de faire passer l'intérêt de leurs patients avant l'intérêt pécuniaire que leur offre la mesure étatique. Selon la Cour, «le médecin prescripteur est tenu, d'un point de vue déontologique, de ne pas prescrire un médicament donné si celui-ci ne convient pas au traitement thérapeutique de son patient, et ce nonobstant l'existence d'incitations financières publiques à la prescription de ce médicament». Au besoin, les autorités de surveillance des médecins veillent au respect de cette déontologie. Enfin, des mesures orientant la prescription médicale s'apparentent à une politique de sécurité sociale, champ réglementaire où les gouvernements nationaux - et non l'Union européenne demeurent compétents.

\section{Les Etats de l'Union européenne sont libres d'offrir des avantages financiers aux médecins acceptant de prescrire les médicaments les moins chers, à condition d'exposer de manière transparente les choix effectués}

La Cour ne laisse cependant pas carte blanche aux Etats pour développer leur système d'assurances sociales. Les mesures nationales, dont celles offrant des incitations financières aux médecins, doivent s'appuyer «sur des critères objectifs» et ne pas opérer de discrimination «entre les médicaments nationaux et ceux provenant d'autres Etats membres». Pour permettre le contrôle de ces exigences, l'Etat qui adopte un tel système d'incitations doit le rendre public. De surcroît, il doit tenir à disposition des médecins et des sociétés pharmaceutiques «les évaluations établissant l'équivalence thérapeutique entre les substances actives disponibles appartenant à la classe thérapeutique faisant l'objet dudit système».

En résumé, les Etats de l'Union européenne sont libres d'offrir des avantages financiers aux médecins acceptant de prescrire les médicaments les moins chers, à condition d'exposer de manière transparente les bases - avant tout scientifiques - sur lesquelles reposent leur appréciation de l'équivalence thérapeutique des médicaments attribués à une même classe [8].

\section{Cette jurisprudence européenne est-elle transposable à la Suisse?}

Ainsi qu'il a été relevé plus haut, la Suisse connaît depuis 2002 une règle très semblable à la disposition communautaire analysée ici par la Cour européenne [9]. Controversé dès son entrée en vigueur, l'article 33 LPTh interdit l'octroi d'avantages matériels aux médecins (entre autres), à l'exception de ceux qui, à la fois sont de valeur modeste et ont un rapport avec la pratique de la médecine; les rabais usuels ou justifiés économiquement sont également admis s'ils sont répercutés sur le prix facturé aux caisses ou aux patients. De même que l'article 94 de la Directive 2001/83, l'article 33 LPTh ne précise pas le cercle des personnes soumises à cette interdiction. Aussi, peut-il théoriquement s'appliquer à des personnes sans lien avec l'industrie pharmaceutique.

Effectivement, Swissmedic (l'autorité compétente en Suisse pour les médicaments) part de l'idée que les règles sur la publicité doivent être respectées aussi par des particuliers - du moins si leurs actions sont susceptibles de promouvoir la vente de médicaments. Il l'a notamment fait savoir dans ses explications relatives à la publicité pour les médicaments sur Internet [10]. S'agissant plus spécifiquement de l'interdiction érigée par l'article 33 LPTh, Swissmedic estime que «[t]oute personne active dans le commerce de médicaments au sens large peut être concernée. Cela a pour conséquence que, par exemple, une agence de publicité ou une assurance sociale peut violer l'art. 33 LPTh en octroyant un avantage illicite» [11].

Dans un arrêt de 2006, suivant en cela Swissmedic, l'ancienne Commission de recours pour les produits thérapeutiques (CORE PT [12]) avait confirmé que les articles 31 et suivants LPTh peuvent également s'appliquer à des caisses-maladie. Pour cette commission, la caisse qui invite ses assurés à demander à leurs médecins le générique meilleur marché, en lieu et place du médicament original coûteux, émet bel et bien une publicité. En effet, toute mesure qui influence un groupe de patients en l'incitant à choisir un médicament donné tombe sous la définition large de la publicité [13]. Selon la jurisprudence constante, l'un des objectifs des normes suisses sur la publicité est de préserver l'indépendance de jugement des médecins [14]. Au vu de cet objectif, la CORE PT avait estimé qu'il se justifiait de soumettre les caisses-maladie aux mêmes règles, puisque leur but était précisément d'influencer les patients, et indirectement les médecins [15]. Le Tribunal fédéral n'a, pour sa part, jamais eu l'occasion de se prononcer sur la question qui n'est pourtant pas évidente, vu la légitimité d'intérêts divergents en jeu.

Pour revenir à la question cruciale des incitations financières, faut-il partir du principe que l'Etat, respectivement les caisses-maladie, tombent dans le champ d'application de l'art. 33 LPTh?

Depuis déjà juillet 2001, il est admis que les caisses peuvent récompenser par des avantages finan- 


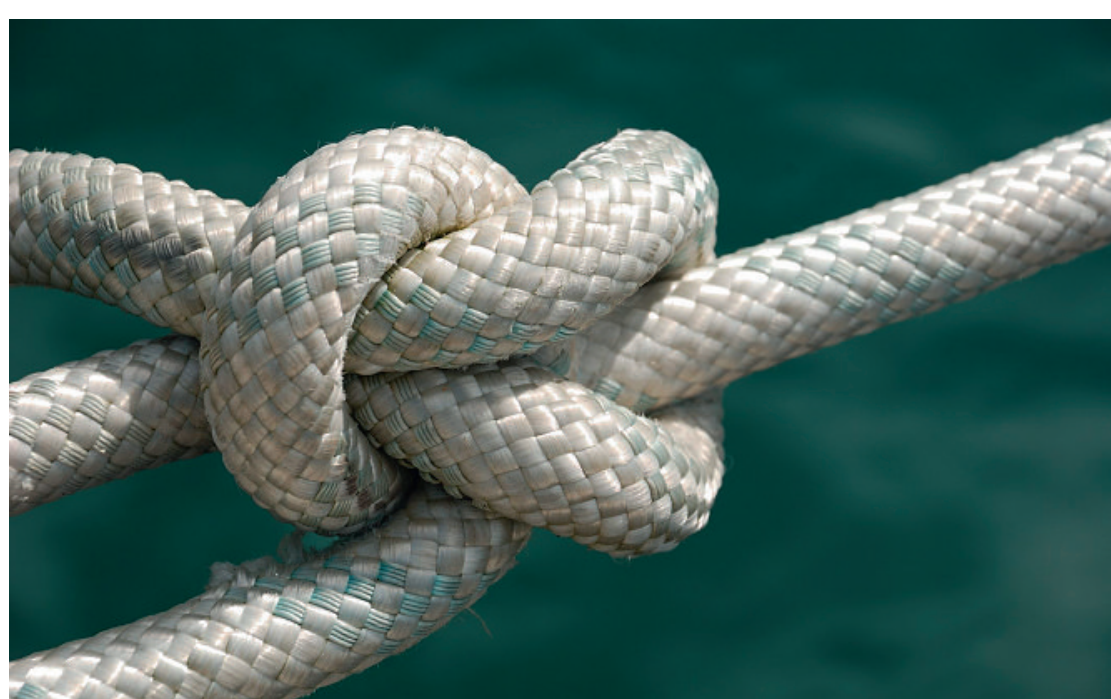

La révision de la LPTh prévoit d'instituer une obligation à charge des médecins de déclarer leurs liens d'intérêts avec les sociétés pharmaceutiques. Mais qu'en est-il de leurs liens avec les caisses-maladie?

\section{Et à I'avenir?}

L'article 33 LPTh a fait l'objet d'un torrent ininterrompu de critiques. Le Conseil fédéral, pressé par le Parlement et certains cantons [19], a finalement mis en ouvre le processus législatif destiné à le réviser [20]. Selon une proposition soumise à la consultation en octobre 2009, l'article 33 LPTh serait abrogé et remplacé par une nouvelle section 2a comportant trois nouvelles dispositions (les articles 57a à 57c) [21]. Les contours de l'interdiction des avantages matériels seraient rendus plus clairs, en précisant que l'avantage n'est interdit que s'il est susceptible d'influencer la prescription, la remise ou l'administration de médicaments soumis à ordonnance [22]. Malheureusement le cercle des personnes soumises à l'interdiction d'offrir de tels avantages n'est pas mieux défini. Bien que l'exécutif soit clairement parti de l'idée que l'interdiction concerne avant tout les sociétés pharmaceutiques, on ne peut exclure que son application englobe d'autres acteurs [23].

C'est pourquoi, dans ce contexte d'incertitude juridique, l'arrêt européen doit être accueilli comme un signal en faveur de l'autonomie de l'Etat et des caisses-maladie. Ce thème pourrait devenir d'une actualité brûlante dans le cadre des discussions sur les réseaux de soins [24]. En effet, la promotion du «managed care» passe par des accords privés entre caisses et médecins. Ces accords visent notamment à optimaliser les dépenses de santé, y compris pour les médicaments. Quand bien même la plus grande opacité règne actuellement sur ces accords, il y a tout lieu de penser qu'ils renferment des incitations financières liées à la prescription de certains médicaments plutôt que d'autres.

\section{Il faut éviter qu'un médicament inutile soit prescrit parce que le médecin en profite financièrement}

Le débat sur les formes que prennent ces incitations mérite d'être mené de manière ouverte. Un incitatif financier peut être bon ou mauvais, selon la manière dont il est conçu [25], d'une part, et surveillé [26], d'autre part. Il faut éviter qu'un médicament inutile soit prescrit parce que le médecin en profite financièrement; il faut éviter que le mauvais médicament soit favorisé sur la base d'études mal comprises ou obsolètes. A l'inverse, peut être tout aussi nuisible le fait de récompenser le médecin pour n'avoir pas prescrit de médicaments, alors même que son patient en a besoin. Trouver le bon équilibre est extrêmement délicat [27]. Les enjeux sont majeurs: santé des patients, équité dans l'accès aux soins, confiance envers les professionnels de la santé, mais aussi stabide la Directive 2001/83. 
lisation des dépenses de santé. L'Etat a clairement un rôle à jouer dans ce débat, mais le public doit pouvoir exercer un contrôle sur les choix étatiques ... tout comme ceux des médecins et des caisses [28]. Le projet de révision de la LPTh prévoit d'instituer l'obliga-

\section{Il faut éviter que le mauvais médicament soit favorisé sur la base d'études mal comprises ou obsolètes}

tion pour les médecins de déclarer leurs liens d'intérêts avec les sociétés pharmaceutiques [29]. Il devrait en aller de même des liens financiers entre médecins et caisses-maladie. Le maintien de l'indépendance des uns et des autres est à ce prix.

\section{Références}

1 Arrêt C-62/09 du 22 avril 2010, disponible à partir de http://curia.europa.eu/fr/content/juris/index.htm. La Cour européenne se prononce ici sur question dite préjudicielle, c'est-à-dire uniquement sur le point de droit communautaire litigieux délimité par le tribunal national.

2 L'arrêt ne précise pas si ces paiements trouvent leur appui dans une loi anglaise; tel n'est apparemment pas le cas. L'arrêt ne dit pas non plus à combien le paiement s'élève. Selon un document trouvé sur Internet, le paiement est calculé en fonction du total de points attribués à de multiples indicateurs, dont fait partie la substitution thérapeutique. S'agissant de la caisse North of Tyne, il est plafonné à $10000 £$ par cabinet (groupe) de médecins. Cf. NHS North of Tyne, Prescribing Incentive Scheme 2009/10 (2009). Selon une étude effectuée en 2000 par Mark Ashworth et al, les montants varient selon les caisses et vont de $3000 £$ à plus de $20000 £$. Cf. Prescribing incentive schemes in two NHS regions: cross sectional survey. 324 British Medical Journal. 2002; p. 1187-8.

3 Selon cette disposition, «[d]ans le cadre de la promotion des médicaments auprès des personnes habilitées à les prescrire ou à les délivrer, il est interdit d'octroyer, d'offrir ou de promettre à ces personnes une prime, un avantage pécuniaire ou un avantage en nature à moins que ceux-ci ne soient de valeur négligeable et n'aient trait à l'exercice de la médecine ou de la pharmacie.» Cf. Directive 2001/83/CE du 6 novembre 2001 instituant un code communautaire relatif aux médicaments à usage humain.

4 Selon cette disposition, «[i]l est interdit d'octroyer, d'offrir ou de promettre des avantages matériels aux personnes qui prescrivent ou remettent des médicaments ainsi qu'aux organisations qui emploient de telles personnes. (...) Sont admis: a. les avantages matériels de valeur modeste et qui ont un rapport avec la pratique de la médecine ou de la pharmacie; b. les rabais usuels dans le commerce et justifiés économiquement qui se répercutent directement sur le prix.»
5 Que l'industrie pharmaceutique s'érige en champion de l'indépendance de jugement des médecins peut prêter à sourire. Cependant, comme le constate l'Avocat général Nilo Jääskinen auprès de la Cour, «le caractère intéressé de la motivation d'ABPI n'est pas juridiquement pertinent. En tant que branche d'activité, l'industrie pharmaceutique est légale, socialement utile et même encouragée par le législateur européen. Il est également inhérent à l'ordre économique de l'Union européenne, qui tend à l'établissement d'un marché ouvert dans lequel la concurrence s'exerce librement, que les opérateurs économiques privés poursuivent des fins lucratives. Cette logique s'applique également à l'industrie pharmaceutique.»

6 Les arguments de l'Avocat général étant particulièrement intéressants, ses conclusions du 11 février 2010 méritent lecture. Pour un commentaire de ces conclusions, voir par ex. Manley MI, Barnden M. Advocate General's Opinion from the ECJ Finds Public Bodies' Prescribing Incentive Schemes to be Unlawful. RAJ Pharma. 2010; p. 215-20.

7 Arrêt C-421/07 «Damgaard» du 2 avril 2009; voir aussi mon commentaire dans Medialex 2/2010 p. 8-14.

8 Il n'est pas sûr que ces conditions soient remplies dans le système anglais de sorte qu'il s'agit de points que le tribunal national devra vérifier. Cependant, pour minimiser les risques liés à ces incitations financières, le Department of Health anglais a émis une directive intitulée «Strategies to achieve cost-effective prescribing - interim guidance for primary care trusts». Y figurent notamment des indications sur les critères objectifs à prendre en compte. Par ailleurs, la directive recommande aux médecins d'utiliser l'argent perçu par leur cabinet à des fins qui soient bénéfiques à leurs patients.

9 Le souci d'harmonisation avec le droit communautaire et d'eurocompatibilité conduit la Suisse à se calquer sur la majorité des règles européennes, tout particulièrement dans le secteur pharmaceutique.

10 Voir la réponse à la question 59 du document «La publicité pour les médicaments sur Internet»: questions posées lors de la séance d'information (novembre/décembre 2006).

11 Cf. Swissmedic. L'interdiction de la promesse et de l'acceptation d'avantages matériels au sens de l'article 33 de la Loi sur les produits thérapeutiques, en particulier l'admissibilité du soutien de la formation postgraduée et continue des médecins par l'industrie pharmaceutique, Journal Swissmedic. 2006;(6):616.

12 En 2007, la CORE PT a été remplacée par le Tribunal administratif fédéral.

13 La jurisprudence allait très loin puisque même le fait de fournir une information objective était réputé relever de la publicité si l'effet était de promouvoir les ventes ( «Selbst die blosse Information über Preise von Arzneimitteln stellt demnach eine Werbung dar, wenn sie bestimmt und geeignet ist, das Konsumverhalten zu beeinflussen.» Considérant 3.1. de l'arrêt HM 05.14). Depuis, cette notion très extensive de la publicité a été quelque peu restreinte. Cf. arrêt du Tribunal fédéral du 13 juin 2007, 2A.787/2006, consid. 5; aussi arrêt du $1^{\mathrm{er}}$ octobre 2008.

2C_93/2008, consid. 4.1.; aussi: Kramer B. Werbung oder Information? Zur Abgrenzungspraxis in der Arzneimittelwerbung. sic. 2007(6):489. 
14 «'Lart. 33 LPTh vise à assurer que seules des considérations médicales président à la prescription et à la remise de médicaments, à l'exception de toute incitation financière, afin de garantir au patient le traitement le plus approprié. Cette disposition a donc pour but la sauvegarde de la santé et constitue ainsi une norme relevant de la politique sanitaire.» Arrêt 2P.169/2006 du 20 septembre 2007, considérant 3.3, parenthèses omises.

15 La campagne de lettres de la caisse-maladie n'avait pu être «sauvée» in extremis que par une interprétation audacieuse de l'article 31 al. 2 LPTh, laquelle disposition donne mandat au Conseil fédéral de fixer «les conditions auxquelles des comparaisons de prix de médicaments soumis à ordonnance peuvent être publiées».

16 Cf. articles 25 al. 2 let. h et 52 a de la Loi fédérale sur l'assurance-maladie (LAMal, RS 832.10) et l'article 4a al. 1 let. c de l'Ordonnance sur les prestations de l'assurance des soins (OPAS, RS 832.112.31).

17 La règle ne s'applique toutefois qu'aux médicaments sur prescription à charge de l'assurance obligatoire. Pour ceux-ci, le pharmacien touche de la caisse au maximum 20 points tarifaires lors de la première substitution générique.

18 En cas de contradiction entre deux dispositions légales, le principe veut que la plus spécifique (la plus précise) ait le pas sur la plus générale.

19 Voir principalement la motion 06.3420 de la Commission de la sécurité sociale et de la santé publique du Conseil des Etats, mais aussi les postulats, questions et interpellations parlementaires 02.3139, 02.3237, 02.3657, 02.3572, 03.308e, $03.1101,05.3016,05.3120,06.410,06.3713$.

20 L'avant-projet de loi révisée et le rapport explicatif du Département fédéral de l'intérieur, tous deux datés d'octobre 2009, sont disponibles à partir de www.admin.ch/ch/f/gg/pc/ind2009.html

21 Le Conseil fédéral ne se montre pas du tout pressé, puisque son message ne devrait être transmis au Parlement qu'en 2012. Aussi la loi modifiée et ses nouvelles ordonnances pourront-elles entrer en vigueur, au mieux, début 2014.

22 A l'avenir, les médicaments non soumis à ordonnance ne seront plus visés par l'interdiction des avantages matériels. Par ailleurs, la référence à l'influence sur la prescription, la remise et l'administration vise à uniformiser la version française avec les versions existantes allemande et italienne; l'influence n'a toutefois pas besoin d'être prouvée.
23 Des pharmaciens ont déjà tenté - en vain - de faire appliquer l'interdiction des avantages matériels à la pharmacie par correspondance Zur Rose, laquelle offre 5 CHF aux médecins qui lui transmettent une ordonnnance électronique. Leurs recours avaient toutefois été déclarés irrecevables, de sorte que la question de l'application de l'article 33 aux pharmacies par correspondance n'a pas été tranchée (cf. arrêts du Tribunal fédéral 2P.32/2006, 2A.56/2006, 2C.803/2008). Pour sa part, Swissmedic avait considéré que l'article 33 n'était pas applicable dès lors que la rémunération offerte aux médecins ne visait qu'à les inciter à utiliser un canal de distribution particulier, et non pas à opter pour certains médicaments plutôt que d'autres.

24 Projet de révision partielle de la LAMal, objet 04.062 .

25 A titre d'exemple, le fait de récompenser financièrement la substitution générique n'engendre normalement aucun risque pour le patient. De même, au sein de certaines classes thérapeutiques (notamment les inhibiteurs de la pompe à protons), on peut imaginer des incitatifs portant sur les molécules disponibles sous forme génériques (par ex. omeprazol, plutôt que esomeprazol). A ce sujet, voir par ex. le numéro 2 du bulletin d'information sur les médicaments de la Commission des médicaments des Hôpitaux universitaires de Genève (HUG) (novembre 2007).

26 Il est bien sûr difficile de déterminer quels sont les effets engendrés par un incitatif financier. A la rigueur, une étude avant-après l'introduction d'un tel incitatif pourrait être menée en recourant aux bases de données des assureurs. Il est cependant nullement certain que ceux-ci acceptent de les mettre à disposition des chercheurs.

27 Les incitatifs financiers devraient également être comparés, sur le plan des bénéfices attendus et des risques envisageables, avec les incitations nonfinancières, telle l'encouragement de la formation universitaire ou continue des médecins.

28 Si une transparence totale des conventions adoptées n'est pas envisageable car elle rendrait impossible ou difficile à l'excès les négociations entre partenaires tarifaires, une information résumée sur les incitations financières convenues est, elle, tout à fait possible.

29 Cf. l'article 57c du projet de révision de la LPTh. 